\title{
Norois
}

Environnement, aménagement, société

193 | 2004/4

Varia

\section{Modes de vie périurbains en France}

Le cas d'une aire urbaine de taille moyenne, La Roche-sur-Yon (Vendée)

Suburban ways of life in France: the case of a middle-sized urban area, La

Roche-sur-Yon (Vendée)

\section{François Madoré}

\section{OpenEdition}

Journals

Édition électronique

URL : http://journals.openedition.org/norois/789

DOI : $10.4000 /$ norois. 789

ISBN : 978-2-7535-1541-3

ISSN : $1760-8546$

Éditeur

Presses universitaires de Rennes

\section{Édition imprimée}

Date de publication : 1 décembre 2004

Pagination : 77-90

ISBN : 978-2-7535-0081-5

ISSN : 0029-182X

Référence électronique

François Madoré, « Modes de vie périurbains en France », Norois [En ligne], 193 | 2004/4, mis en ligne le 18 août 2008, consulté le 01 mai 2019. URL : http://journals.openedition.org/norois/789 ; DOI :

10.4000/norois.789

Ce document a été généré automatiquement le 1 mai 2019.

(c) Tous droits réservés 


\section{Modes de vie périurbains en France}

Le cas d'une aire urbaine de taille moyenne, La Roche-sur-Yon (Vendée)

Suburban ways of life in France: the case of a middle-sized urban area, La

Roche-sur-Yon (Vendée)

François Madoré

\section{NOTE DE L'ÉDITEUR}

Cet article a été reçu le 14 juin 2004 et définitivement accepté le 28 septembre 2004.

\section{Introduction}

1 Le schéma de mobilité quotidienne des ménages vivant en milieu urbain a été profondément modifié au cours des dernières décennies en France, du fait, en particulier, d'un contraste majeur survenu dans l'évolution de l'organisation urbaine. Un phénomène de dissociation relative entre, d'une part, la croissance démographique des aires urbaines et, d'autre part, la localisation de l'emploi au sein de ces entités est observé. La géographie résidentielle est ainsi marquée par la vigueur du processus de périurbanisation, qui marque la fin de la ville européenne dense et compacte. En revanche, "la périurbanisation de l'emploi est moins prononcée ", même si le mouvement de diffusion centrifuge des activités économiques est également engagé, en particulier dans «les grandes aires urbaines de province les plus dynamiques » (Lainé, 2000). Ce mouvement contrasté des composants de la structure urbaine alimente tout un discours et de nombreux écrits sur la ville, dont la floraison de néologismes traduit la difficulté de conceptualisation, qu'il s'agisse de la «métapole » (Ascher, 1995), de la « ville émergente » (Dubois-Taine et Chalas, 1997) ou encore de la "ville éclatée » (May et al., 1998; Haumont et Lévy, 1998). C'est donc l'ensemble des pratiques spatiales qui se trouvent modifiées par ce découplage relatif entre résidence et emploi, tout 
particulièrement pour la population périurbaine, qui est au cœur de ce processus de dissociation (Le Guirriec, 1997 ; Madoré, 1998).

2 Afin d'étayer et de vérifier la pertinence de cette énonciation, les pratiques spatiales de la population périurbaine vont être observées, en cherchant à vérifier une double hypothèse. Certes, de nombreux travaux visant à mieux comprendre le schéma de mobilité dite quotidienne des périurbains ont déjà été réalisés, en particulier au cours des années quatre-vingt ${ }^{1}$, mais il nous semble que les deux hypothèses qui sous-tendent notre réflexion n'y étaient pas véritablement posées de façon explicite. La première de ces hypothèses est que les pratiques citadines mises en œuvre par la population périurbaine, à travers l'effet de mobilité à destination du pôle urbain, s'accompagnent d'un processus de "périphérisation » des modes de vie, autrement dit d'une fréquentation plus portée vers les polarités périphériques que vers le cœur de la ville, par comparaison avec celle des habitants des pôles urbains. La seconde hypothèse est que ce rapport à l'espace urbain est toujours guidé pour partie par la recherche de la proximité, car certaines pratiques participent d'un ancrage dans la commune de résidence.

\section{Méthodologie}

3 Afin d'observer la dynamique de périurbanisation en France, l'Insee a mis au point, au milieu des années quatre-vingt-dix, une nouvelle approche territoriale de la ville, fondée sur le zonage en aires urbaines (ZAU) (Le Jeannic, 1996). Celui-ci délimite en particulier des aires urbaines composées d'un pôle urbain et d'une couronne périurbaine (fig. 1). Les pôles sont des unités urbaines offrant plus de 5000 emplois, sous réserve qu'elles ne soient pas sous la dépendance directe d'un pôle urbain plus important. Quant aux couronnes périurbaines, elles sont composées des communes où au moins $40 \%$ de la population active résidente ayant un emploi travaille dans le pôle urbain ou dans les autres communes de la couronne périurbaine. L'intérêt de ce découpage en aires urbaines est qu'il légitime statistiquement l'identité urbaine des communes marquées par le développement, depuis les années soixante-dix, de la périurbanisation, et qu'il offre de ce fait un cadre d'observation pertinent de la réalité des pratiques sociales en milieu urbain. C'est donc à l'échelle de ces aires urbaines que sera analysé ce phénomène de distanciation entre la résidence, qui se dilue dans les franges périurbaines, et l'emploi, dont la concentration dans les aires urbaines reste nettement plus marquée. 
Figure 1 : La structuration urbaine de La Roche-sur-Yon en 2000 Urban pattern of La Roche-sur-Yon in 2000

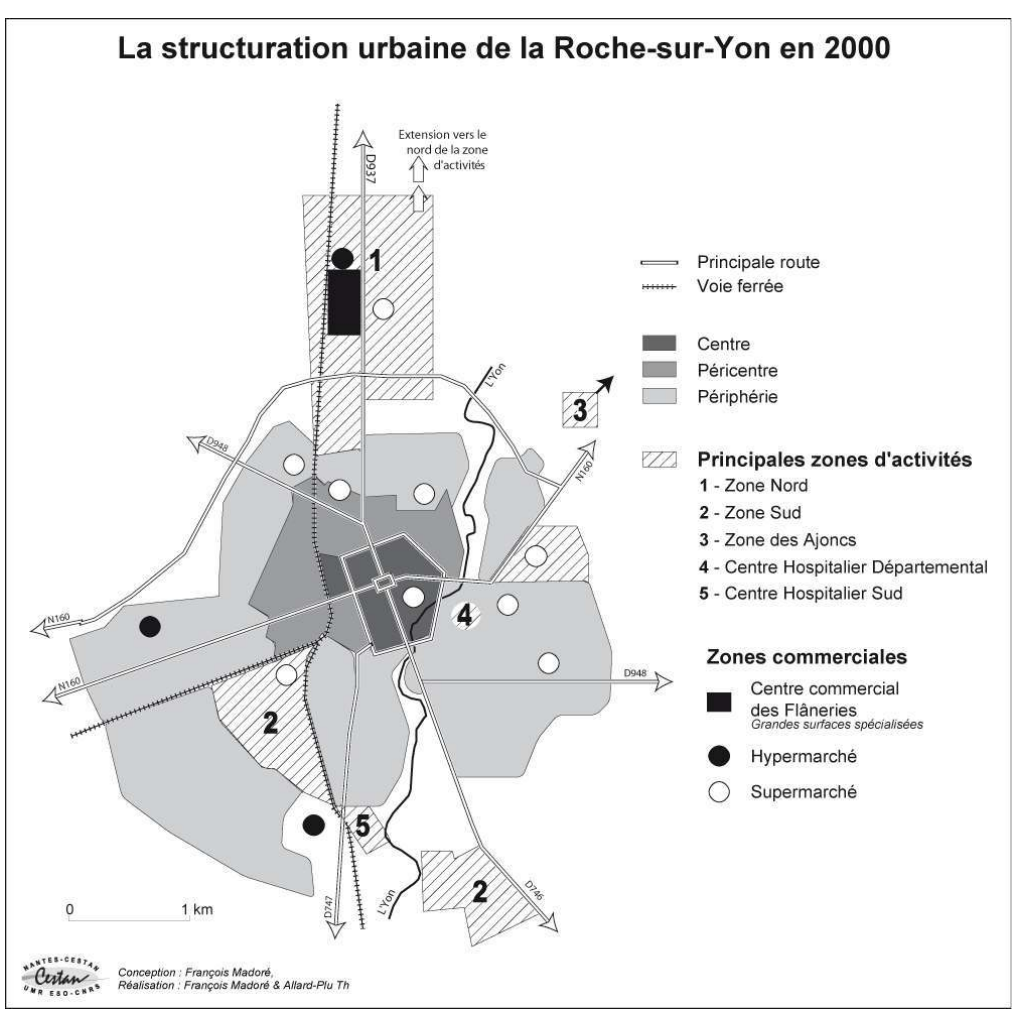

4 Quant à l'observation des pratiques spatiales de la population périurbaine, elle passe nécessairement par la mobilisation de l'enquête par questionnaire. C'est le moyen idoine pour appréhender, avec beaucoup de finesse, la diversité des schémas de mobilité mis en œuvre par la population et pour vérifier la double hypothèse d'une "périphérisation " des modes de vie périurbains et du maintien de la recherche de proximité dans certaines pratiques de l'espace. D'où l'intérêt d'une approche monographique éclairée et mise en perspective par cette problématisation. Le matériau de recherche est donc constitué d'une enquête financée par la ville de La Roche-sur-Yon en Vendée, dans le but de saisir, à l'échelle de cette aire urbaine, les comportements de mobilité autres que ceux de nature résidentielle et la représentation de la centralité liée à ces pratiques. 1000 enquêtes ont été réalisées en 2000 auprès de la population de l'aire urbaine, telle qu'elle a été définie par l'Insee en $1990^{2}$. Celle-ci était composée, à l'issue de ce recensement, d'un pôle urbain constitué d'une ville isolée de 49262 habitants en 1999 et d'une couronne périurbaine rassemblant vingt communes et 44624 habitants, soit 93886 habitants pour l'aire urbaine (avec le zonage en aire urbaine de 1999, la couronne périurbaine a gagné quatre communes et atteint désormais 48913 habitants). L'échantillon retenu pour cette enquête est représentatif de l'ensemble de la population occupant une résidence principale, les quotas ayant été établis en tenant compte du lieu de résidence en 1999, puis du sexe et de l'âge en $1990^{3}$. L'objectif étant d'observer les pratiques spatiales d'une population périurbaine composée en majorité de couples biactifs ayant des enfants, ce sont les réponses données par les 475 individus enquêtés dans la couronne périurbaine qui vont être plus particulièrement exploitées. Trois types de pratiques vont retenir l'attention, car elles sont au cœur de la mobilité et du mode de vie des individus : les pratiques liées à 
l'emploi, celles articulées autour de la fréquentation des commerces et enfin les pratiques commandées par le recours aux services privés de base.

Bien évidemment, l'adoption de la démarche monographique contient une mise en garde implicite, mais qu'il faut néanmoins rappeler, afin d'éviter tout risque de généralisation abusive. Chaque aire urbaine étant singulière, celle de La Roche-sur-Yon, par sa taille réduite, ne peut donc être considérée comme représentative de l'ensemble des aires urbaines françaises, a fortiori des plus peuplées. Toutefois, l'intérêt de la démarche tient à la mobilisation d'un vaste matériau d'enquêtes susceptible d'éclairer empiriquement certaines tendances des modes de vie périurbains et d'apporter ainsi un contrepoint à d'autres observations.

\section{Desserrement résidentiel et maintien d'une localisation centrale de l'emploi dans les aires urbaines françaises}

\section{La poursuite de la croissance urbaine et l'émergence de la périurbanisation \\ à partir des années soixante-dix}

6 L'essor de l'urbanisation à partir de la seconde moitié du XIX ${ }^{e}$ siècle a permis à la France d'enregistrer un mouvement de concentration spatiale sans précédent de son peuplement. Toutefois, si le modèle historique de la ville dense reste la référence jusque dans les années soixante, un phénomène nouveau est apparu au cours de la décennie soixante-dix. En effet, parallèlement au ralentissement de la croissance démographique en France, les unités urbaines ont vu leur population stagner au profit des communes proches des villes, ce déplacement aux franges des unités urbaines des zones dynamiques démographiquement donnant naissance à la périurbanisation. C'est d'ailleurs pour rendre compte de ce phénomène que l'Insee a adopté le zonage en aires urbaines, qui permet de bien lire les variations spatiales des évolutions démographiques, contrairement au concept de l'unité urbaine, qui reste fondé sur le continuum d'urbanisation.

7 À la lumière de ce zonage, deux faits majeurs méritent d'être soulignés, pour la période récente. D'une part, la poursuite de l'urbanisation ne s'est jamais démentie en France tout au long des dernières décennies, le recensement de 1999 confirmant cette tendance (Julien, 2001). En raisonnant à espace constant, c'est-à-dire en se référant à la définition des aires urbaines en 1990, l'intégralité des gains démographiques enregistrés en France depuis les années soixante est le fait de l'espace à dominante urbaine, dont la population a augmenté au rythme annuel moyen de $+0,85 \%$, alors que celle de l'espace rural a été remarquablement stable à 13,6 millions d'habitants (tableau 1). 
Tableau 1 : L'évolution de la population de l'espace à dominante urbaine et rurale en France de 1962 à 1999 (aires urbaines 1990)

The evolution of population in urban and rural space in France between 1962 and 1999 (urban areas 1990)

\begin{tabular}{|c|c|c|c|c|c|}
\hline \multirow{2}{*}{ Type d'espace } & \multicolumn{2}{|c|}{1962} & \multicolumn{2}{c|}{1999} & $1962-1999$ \\
\cline { 2 - 6 } & $\begin{array}{c}\text { effectif } \\
\text { (en milliers) }\end{array}$ & en \% & $\begin{array}{c}\text { effectif } \\
\text { (en milliers) }\end{array}$ & en \% & $\begin{array}{c}\text { en \% annuel } \\
\text { moyen }\end{array}$ \\
\hline & & & & & 74891 \\
Espace à dominante urbaine & 32812 & 71 & 44 & $+0,85$ \\
Espace à dominante rurale & 13613 & 29 & 13627 & 23 & 0,00 \\
\hline France métropolitaine & 46425 & 100 & 58518 & 100 & $+0,63$ \\
\hline
\end{tabular}

(source: Insee, recensements de la population 1962 et 1999)

8 D'autre part, cette poursuite de l'urbanisation a pris une nouvelle dimension au milieu des années soixante-dix, avec la dynamique de périurbanisation. Toujours en se référant à la définition des aires urbaines françaises en 1990, le rythme d'évolution annuelle de la population des couronnes périurbaines a été plus de deux fois supérieur à celui des pôles urbains entre 1962 et 1999, avec respectivement $+1,64 \%$ contre $+0,71 \%$ (tableau 2). Un examen plus attentif montre la rupture survenue au milieu desannées soixante-dix (Le Jeannic, 1997). En effet, au cours de la décennie soixante, les pôles urbains enregistrent une forte progression démographique, qui se prolonge tout en s'atténuant au cours de la période 1968-1975. En revanche, la période qui s'ouvre à partir de 1975 est marquée par une nette décélération du rythme d'évolution de ces pôles et l'émergence de la périurbanisation. Ainsi, le taux d'évolution annuel des pôles urbains est divisé par cinq entre 1962-1975 et 1975-1990 (+1,48\% à + 0,30\%), alors que dans le même temps ce taux est multiplié par $1,7(+1,34 \%$ à $+2,28 \%)$ pour les couronnes périurbaines. La hiérarchie des taux s'est donc complètement inversée en peu de temps, la population des couronnes périurbaines augmentant quasiment huit fois plus vite que celle des pôles urbains entre 1975-1990. Quant à la dernière période intercensitaire, elle n'apporte aucune rupture dans ces rythmes d'évolution pour les pôles urbains, dont la population continue à croître lentement $(+0,27 \%)$. En revanche, elle amplifie la poursuite du ralentissement de la périurbanisation déjà amorcé entre 1982-1990 (Choffel, 2000), puisque le rythme d'évolution annuel de la population des couronnes périurbaines, après avoir atteint son paroxysme entre $1975-1982$ avec $+2,71 \%$, recule à $+1,89 \%$ entre $1982-1990$, pour de nouveau diminuer entre 1990-1999 et s'établir à $+1,03 \%$. Toutefois, les couronnes périurbaines continuent à progresser encore quatre fois plus vite que les pôles urbains et le ralentissement de la dynamique de périurbanisation, certes très sensible en taux, l'est beaucoup moins en effectifs. 
Tableau 2 : L'évolution de la population des pôles urbains et de leurs couronnes périurbaines en France de 1962 à 1999 (aires urbaines 1990)

The evolution of population in urban centre and surrounding suburbs in France between 1962 and 1999 (urban areas 1990)

\begin{tabular}{|c|c|c|c|c|}
\hline \multirow[b]{2}{*}{ Type d'espace } & $1962-1975$ & $1975-1990$ & $1990-1999$ & $1962-1999$ \\
\hline & $\begin{array}{c}\text { en } \% \text { annuel } \\
\text { moyen }\end{array}$ & $\begin{array}{c}\text { en } \% \text { annuel } \\
\text { moyen }\end{array}$ & $\begin{array}{c}\text { en } \% \text { annuel } \\
\text { moyen }\end{array}$ & $\begin{array}{c}\text { en } \% \text { annuel } \\
\text { moyen }\end{array}$ \\
\hline Pôles urbains & $+1,48$ & $+0,30$ & $+0,27$ & $+0,71$ \\
\hline Couronnes périurbaines & $+1,34$ & $+2,28$ & $+1,03$ & $+1,64$ \\
\hline Rapport couronnes / pôles & 0,91 & 7,60 & 3,81 & 2,31 \\
\hline
\end{tabular}

(source : Insee, recensements de la population 1962 à 1999)

\section{La nature du processus de périurbanisation : desserrement résidentiel...}

9 La poursuite du dynamisme démographique des aires urbaines en France est donc moins le fait, depuis 1975, des pôles urbains, dont la progression est faible, que des couronnes périurbaines, qui enregistrent une forte augmentation de leur population, bien que ralentie depuis 1982. L'existence d'un solde migratoire légèrement négatif dans les pôles depuis 1975, mais fortement positif dans le périurbain (supérieur à $+1 \%$ par an entre 1975-1990, voire à + 2 \% entre 1975-1982), montre que la croissance de ces auréoles a été alimentée par des flux migratoires en provenance des pôles urbains, même si l'importance de la mobilité interne au périurbain n'est pas à négliger, comme l'a bien montré M. Berger (1990) à travers le cas de l'île-de-France. Ainsi, l'essence urbaine de la périurbanisation ne fait pas de doute, ce phénomène n'étant en aucun cas révélateur d'une renaissance rurale. Même les chercheurs qui ont eu recours à un autre néologisme d'origine anglo-saxonne pour qualifier ce processus, en parlant de rurbanisation, dont la terminologie dit combien le sens profond du phénomène serait à chercher à la croisée de rural et de l'urbain, ont tendance, pour la plupart, à considérer ce fait géographique comme «l'une des modalités spatiales de la croissance urbaine » (Berger et al., 1980). D'ailleurs, il nous semble que l'usage équivoque de ce concept de rurbanisation a progressivement périclité au profit de celui de périurbanisation, sémantiquement plus proche de la signification socio-spatiale du phénomène. Sans doute faut-il voir dans cette substitution une forme de légitimation du postulat selon lequel la périurbanisation résulte bien du « développement d'une forme urbaine adaptée à l'état momentané des forces productives et des rapports sociaux » (Jaillet et Jalabert, 1982), postulat qui semble faire l'unanimité, ou peu s'en faut (Taffin, 1985 ; Chapuis, 1995 ; Jean et Calenge, 1997 ; Le Jeannic, 1997) ${ }^{4}$.

Quel est cet "état momentané des forces productives et des rapports sociaux 》 évoqué par M.-C. Jaillet et G. Jalabert en 1982, qui recompose le système urbain à partir des années soixante-dix en France? Celui-ci relève d'abord des transformations de l'expression de la demande en logements des ménages. L'attente des Français, dans ce domaine, se traduit à la fois par un rejet des formes précédentes de production de la trame urbaine inspirées du fonctionnalisme et par l'émergence de revendications plus qualitatives, dont l'une des modalités est la volonté d'accéder à la propriété d'un pavillon. 
Toutes les recherches conduites sur le périurbain ont bien montré que cet essor de la périurbanisation est associé à l'évolution du cycle familial et patrimonial des ménages, en particulier au double souhait de posséder un plus grand logement lorsque la famille s'agrandit et de devenir propriétaire d'une maison individuelle (Madoré, 1998). Ce parcours résidentiel dit ascendant renvoie au statut valorisant, tant socialement que symboliquement, de la propriété d'occupation, en particulier en habitat individuel. D'autre part, cette diffusion du modèle pavillonnaire en milieu périurbain n'a été rendu possible que par la généralisation de l'usage de l'automobile, qui «ouvre à chacun un vaste espace d'arbitrage en matière de localisation et d'enracinement résidentiel» (Orfeuil, 1995).

11 Cependant, si l'essor de l'accession à la propriété semble consubstantiel au développement périurbain, ne négligeons pas l'évolution de la structure de l'offre. P. Bourdieu (1990) a bien souligné, à juste titre, combien « il est sans doute peu de marchés qui, autant que celui de la maison, soient non seulement contrôlés mais véritablement construits par l'État ». Jamais la demande d'accession à un pavillon périurbain n'aurait pu s'exprimer avec autant de vigueur sans la mise en place de politiques d'encouragement à la propriété à partir des années soixante et soixante-dix (Heugas-Darraspen, 1994; Fribourg, 1998; Vorms, 1998). L'une des figures emblématiques de ces politiques est l'instauration en 1977 du Prêt aidé à l'accession à la propriété (PAP) couplé à l'Aide personnalisée au logement (APL), qui a permis de solvabiliser de nombreux ménages modestes. Or, pour ces derniers, la seule alternative réaliste, compte tenu de la diminution de la rente foncière depuis le cœur du pôle urbain vers sa périphérie, a été de s'éloigner de la ville pour faire construire (Allain, 1992).

\section{...Mais moindre diffusion de l'emploi et des équipements dans les couronnes périurbaines}

12 Parallèlement à cette déconcentration des lieux de résidence à l'échelle des aires urbaines, la force d'attraction économique des pôles urbains s'est globalement maintenue, même si la géographie de l'emploi des agglomérations françaises a également été affectée par un mouvement centrifuge au profit des communes de banlieue mais aussi des couronnes périurbaines. À l'échelle des aires urbaines françaises délimitées en 1990, la concentration de l'emploi dans les pôles urbains est ainsi passée de $90 \%$ en 1962 à $92 \%$ en 1999, alors que, dans le même temps, le poids démographique de ces pôles s'est affaibli de cinq points, régressant de $87 \%$ à $82 \%$. Bien évidemment, le choix de retenir comme espace de référence les aires urbaines en 1990 occulte quelque peu la réalité de la diffusion centrifuge de l'emploi, dans la mesure où les pôles urbains ainsi définis comprennent des communes qui n'étaient pas encore incluses dans ce pôle au cours des années soixante. Si l'on raisonnait avec un référentiel spatio-temporel antérieur, nul doute que l'on constaterait un déplacement plus net de l'emploi vers les périphéries urbaines, comme l'observe F. Lainé (2000). Néanmoins, malgré cette difficulté à apprécier de façon diachronique les dynamiques spatiales de la géographie économique des aires urbaines, il n'en demeure pas moins que le processus de périurbanisation concerne plus l'habitat que l'emploi.

13 Par ailleurs, l'inventaire communal réalisé en 1998 par l'Insee montre bien le souséquipement très marqué des communes périurbaines prises dans leur totalité, c'est-à-dire englobant également les communes multipolarisées ${ }^{5}$, dont la population en 1990 pèse 
pour un cinquième des communes périurbaines. 48 équipements ont été retenus pour hiérarchiser les communes, du fait de leur attraction sur la population des communes voisines qui en sont dépourvues. Ils ont été classés en trois groupes, selon leur zone d'influence. Le premier groupe est composé des six équipements de centres urbains, le second des vingt-six équipements de bourgs-centres et le troisième des seize équipements de villages-centres. Or, on observe "le sous-équipement relatif des communes périurbaines en équipements de bourgs-centres » (Schmitt et Perrier-Cornet, 1998), définis comme les équipements intermédiaires. En effet, une proportion infime (4\%) de communes périurbaines possèdent plus de la moitié des équipements de bourgs-centres, soit moitié moins que les communes du rural isolé $(8 \%)$ ou celles appartenant au rural sous faible influence urbaine $(7 \%)^{6}$ : c'est dire si « le sous-équipement relatif » évoqué par l'Inra et l'Insee apparaît comme un doux euphémisme (tableau 3). C'est d'un véritable désert dont il s'agit, surtout que les communes périurbaines sont, en moyenne, plus peuplées que celles du rural sous faible influence urbaine ou des périphéries des pôles ruraux, avec respectivement 850, 570 et 460 habitants en 1990. Quant aux équipements des villages-centres, définis comme les équipements de proximité, ils ne sont présents de façon significative que dans un quart des communes périurbaines : $24 \%$ d'entre elles seulement possèdent plus de la moitié de ces équipements. C'est, peu ou prou, la proportion observée également dans le rural sous faible influence urbaine et dans le rural isolé.

Tableau 3 : Le niveau d'équipement des communes selon leur appartenance à l'espace à dominante urbaine et rurale en France en 1998 (aires urbaines 1990)

The level of equipment of the communes according to their membership of urban and rural space in France in 1998 (urban areas 1990)

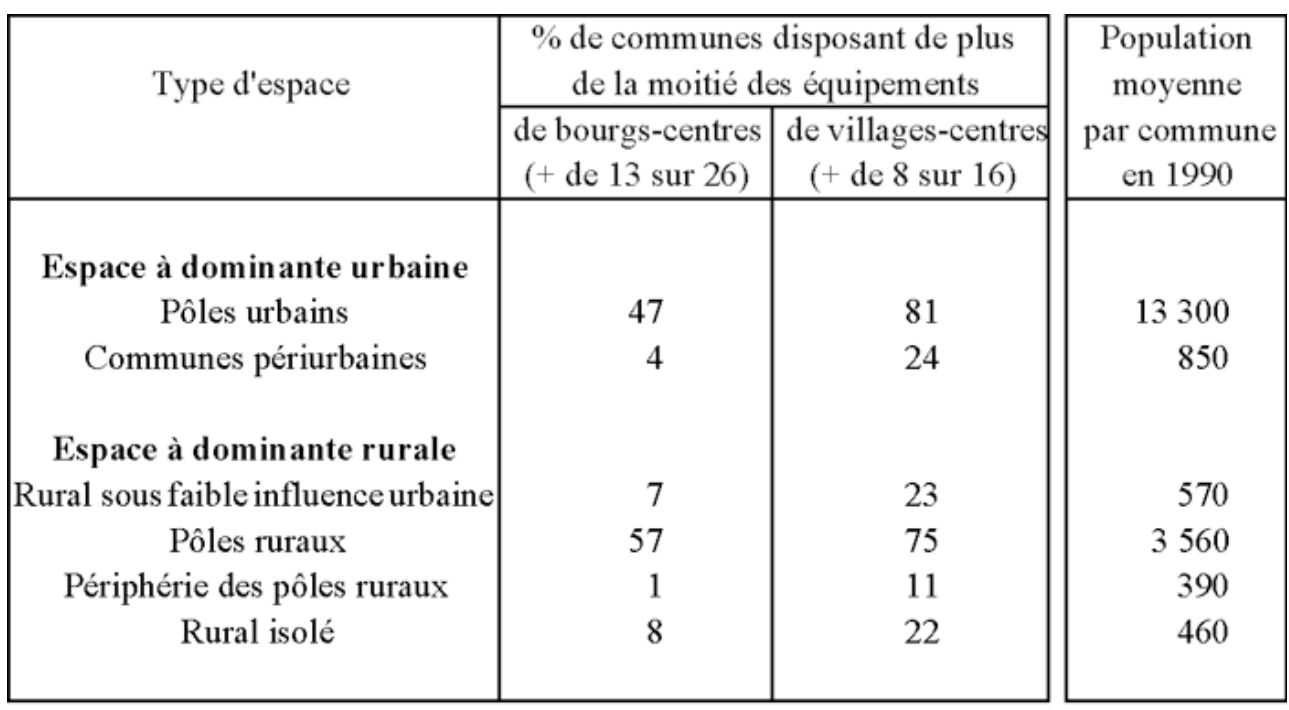

(source : Insee, Inventaire communal, 1998)

À l'évidence, la nature du processus de périurbanisation apparaît clairement dans cette succession de données statistiques, qui permet de brosser avec toute l'objectivité requise un tableau de la situation: il s'agit pour l'essentiel d'un phénomène d'étalement résidentiel, l'emploi, que celui-ci relève de la sphère productive ou de la reproduction, étant moins concerné par ce processus. Ce constat permet donc d'inférer l'hypothèse selon laquelle «la proximité des pôles urbains et l'importance des migrations entre les pôles et leur couronne contribuent probablement au sous-équipement relatif des 
communes périurbaines » (Schmitt et Perrier-Cornet, 1998), ce qui légitime pleinement notre questionnement portant observation des pratiques spatiales ou des modes de vie périurbains, à partir du cas de La Roche-sur-Yon.

\section{Les modes de vie périurbains à La Roche-sur-Yon: entre mobilité, proximité et "périphérisation »}

\section{Les pratiques spatiales des périurbains}

15 Selon le découpage 1990 de l'aire urbaine de La Roche-sur-Yon, la population du pôle urbain, dont les limites géographiques ont été pérennes d'un recensement à l'autre ${ }^{7}$, n'a augmenté que de $10 \%$ entre 1975 et 1999, alors que celle de la couronne périurbaine a cru de plus de moitié : le poids démographique du pôle s'est donc affaibli de neuf points (tableau 4). En revanche, pour l'emploi, les évolutions ont été sensiblement identiques, avec une progression respective de $+45 \%$ et $+37 \%$, si bien que le pôle concentre toujours plus des trois quarts de l'emploi de l'aire urbaine. Du fait de ce découplage entre une périurbanisation vigoureuse de l'habitat et, par contraste, le maintien de la concentration relative de l'emploi dans le pôle urbain, la sur-représentation de l'emploi par rapport à la population atteint 26 points en faveur du pôle en 1990, contre 16 points en 1975.

Tableau 4 : L'évolution de la population et de l'emploi du pôle urbain et de la couronne périurbaine de La Roche-sur-Yon de 1975 à 1999 (aire urbaine 1990)

The evolution of population and employment in urban centre and surrounding suburbs in La Roche-surYon between 1975 and 1999 (urban area 1990)

\begin{tabular}{|c|c|c|c|c|c|c|c|c|c|}
\hline \multirow{2}{*}{ Type d'espace } & \multicolumn{3}{|c|}{ Population } & \multicolumn{4}{c|}{ Emploi } \\
\cline { 2 - 10 } & \multicolumn{2}{|c|}{1975} & \multicolumn{2}{c|}{1999} & \multicolumn{2}{c|}{1975} & \multicolumn{2}{c|}{1999} \\
\cline { 2 - 10 } & effectif & en \% & effectif & en \% & effectif & en \% & effectif & en \% \\
\hline \multirow{3}{*}{ Pôle urbain } & & & & & & & & \\
Couronne périurbaine & 44713 & 61 & 49262 & 52 & 24179 & 77 & 35100 & 78 \\
& 28407 & 39 & 44624 & 48 & 7359 & 23 & 10118 & 22 \\
\hline Aire urbaine & 73120 & 100 & 93886 & 100 & 31538 & 100 & 45218 & 100 \\
\hline
\end{tabular}

(source : Insee, recensement de la population 1975 et 1999)

Parmi les trois variantes de pratiques spatiales prises en compte pour apprécier le mode de vie des habitants de la couronne périurbaine de La Roche-sur-Yon ${ }^{8}$, celles liées aux achats sont les seules à être massivement orientées vers le pôle urbain. Pour $84 \%$ des ménages périurbains, les achats en grande surface sont généralement effectués dans l'un des trois hypermarchés ou l'un des neuf supermarchés yonnais ${ }^{9}$. Cette fréquentation des commerces du pôle urbain s'élève même aux neuf dixièmes pour les achats anomaux, mesurés à partir des achats de vêtements et de livres (tableau 5). Ces pratiques ne se différencient guère de celles développées par les habitants du pôle urbain, montrant bien l'uniformisation des comportements de consommation, appréciés en termes de lieux d'achat, entre les périurbains et ceux qui résident dans le pôle urbain.

Les lieux d'emploi des habitants de la couronne périurbaine de La Roche-sur-Yon sont également orientés, en majorité, vers le pôle urbain, même si celui-ci n'a plus l'exclusivité, comme pour les achats, des destinations. En effet, parmi les actifs ayant un emploi interrogés dans cette couronne périurbaine, 54 \% travaillent à La Roche-sur-Yon, 
contre seulement $21 \%$ dans leur commune de résidence et globalement $29 \%$ pour l'ensemble de la couronne (tableau 5). Le taux de migrants alternants est également très élevé, puisqu'il atteint les quatre cinquièmes. Ce résultat était attendu, car une forte proportion de migrations alternantes est consubstantielle au développement de la dynamique de périurbanisation, étant donné la nature même du phénomène, qui concerne essentiellement la résidence et peu l'emploi. L'Insee montre bien, à partir du recensement de 1999, que « les actifs vivant dans les couronnes périurbaines sont les plus mobiles : 79,1\% d'entre eux changent de commune pour aller travailler» (Talbot, 2001), soit exactement le taux observé dans la couronne périurbaine de La Roche-sur-Yon, à partir de l'enquête réalisée auprès des 475 individus.

En revanche, pour les services privés de base, les habitants de la couronne périurbaine fréquentent faiblement, voire rarement les services de La Roche-sur-Yon. Seulement un quart des périurbains ont leur coiffeur dans le pôle urbain, le taux de fréquentation tombant même à un dixième environ pour la pharmacie ou le médecin généraliste (tableau 5). Ce sont les services situés dans les communes de la couronne périurbaine qui, pour l'essentiel, subviennent aux besoins de la population périurbaine, préférentiellement ceux de la commune de résidence à hauteur de la moitié ou des deux tiers.

Tableau 5 : Les pratiques spatiales (achats, emploi, services) des habitants de la couronne périurbaine de La Roche-sur-Yon en 2000

The space practices (purchase, employment, services) of inhabitants living in surrounding suburbs of La Roche-sur-Yon in 2000

\begin{tabular}{|c|c|c|c|c|c|}
\hline \multirow{4}{*}{$\begin{array}{l}\text { Type de pratiques } \\
\text { spatiales }\end{array}$} & \multicolumn{4}{|c|}{ Communes fréquentées } & \multirow{3}{*}{$\begin{array}{c}\text { Total } \\
\text { répondants }\end{array}$} \\
\hline & \multirow[b]{2}{*}{ Pôle urbain } & \multicolumn{2}{|c|}{ Couronne périurbaine } & \multirow{2}{*}{$\begin{array}{c}\text { Commune situées } \\
\text { hors de l'aire } \\
\text { urbaine }\end{array}$} & \\
\hline & & $\begin{array}{l}\text { Commune de } \\
\text { résidence }\end{array}$ & $\begin{array}{c}\text { Autres } \\
\text { commune }\end{array}$ & & \\
\hline & en $\%$ & en $\%$ & en $\%$ & en $\%$ & effectif \\
\hline Achats & & & & & \\
\hline Grande surface & 84 & 17 & 4 & 11 & 473 \\
\hline Vêtements & 90 & 3 & 1 & 20 & 472 \\
\hline Livres & 93 & 1 & 1 & 5 & 357 \\
\hline Emploi & 54 & 21 & 8 & 17 & 343 \\
\hline Services & & & & & \\
\hline Coiffeur & 27 & 44 & 8 & 9 & 459 \\
\hline Pharmacie & 14 & 65 & 20 & 6 & 473 \\
\hline Médecin généraliste & 10 & 59 & 22 & 9 & 471 \\
\hline
\end{tabular}

(source : enquête auprès d'un échantillon de 1000 habitants de l'aire urbaine de La Roche-sur-Yon, dont 475 de la couronne périurbaine, 2000)

Possibilité de réponses multiples dans certains cas : le total en ligne peut donc être supérieur à 100 . Par ailleurs, pour le coiffeur, il faut compter également avec le domicile (12\%)

(Le résultat du test de Khi ${ }^{2}$ à $99 \%$ est très significatif pour les achats en grande surface et de vêtements, pour le lieu d'emploi, pour la fréquentation du salon de coiffure, de la pharmacie et du médecin généraliste. Il est significatif pour les achats de livres)

19 Au total, parmi les trois types de pratiques spatiales retenus, deux sont structurés par un effet de mobilité indéniable à destination du pôle urbain (l'emploi et de façon encore plus nette les achats), alors que le troisième est au contraire marqué par un ancrage dans la commune de résidence (services privés de base). Cette pratique différenciée de l'espace 
traduit pour partie la répartition très contrastée de l'offre, car à la concentration du commerce et de l'emploi dans le pôle urbain s'oppose la diffusion beaucoup plus large des services privés de base dans la couronne périurbaine. Une seule commune périurbaine sur les vingt possède une librairie et trois seulement ont une grande surface et un commerce de vêtements en 2000, alors que douze ont une pharmacie, quinze un médecin généraliste et dix-huit un salon de coiffure. Aussi, avec une offre commerciale marginale dans la couronne périurbaine, alors que celle-ci rassemble près de la moitié de la population, les pratiques d'achat des périurbains obéissent pour l'essentiel à la force d'attraction commerciale du pôle yonnais. Néanmoins, ces pratiques, pour autant qu'elles soient tournées vers le pôle urbain, sont également structurées par un effet de proximité géographique avec le domicile, révélant la quête d'une optimisation de l'usage du temps (Desse, 2001). Ainsi, les périurbains, dans leur choix d'une grande surface à La Roche-surYon, optent généralement pour celle qui est la plus proche de leur domicile. En revanche, le développement des services basiques ayant suivi le déplacement du centre de gravité démographique du cœur de l'aire urbaine vers ses franges, leur fréquentation obéit pour l'essentiel à un principe de proximité spatiale avec le domicile, ce qui réduit considérablement la mobilité à destination du pôle urbain de La Roche-sur-Yon.

\section{L'influence du lieu de résidence antérieur sur les pratiques spatiales des périurbains}

20 Plusieurs paramètres peuvent avoir une influence sur les pratiques spatiales des périurbains. L'incidence de trois variables a été mesurée pour la fréquentation des commerces et des services privés de base, car elles sont susceptibles d'avoir une incidence sur ces pratiques : il s'agit du lieu de travail des habitants de la couronne périurbaine, de la distance entre leur commune de résidence et le pôle urbain et de leur lieu de résidence antérieur.

21 Pour les deux premières variables, les tests $\mathrm{du} \mathrm{khi}^{2}$ sont peu significatifs, ce qui veut dire que le lieu de travail des périurbains et la distance entre leur commune de résidence et le pôle urbain n'a qu'une influence limitée sur leurs pratiques d'achat et de fréquentation des services. Ce résultat apporte une double confirmation: d'une part, la fréquentation massive des commerces du pôle urbain yonnais par les habitants de la couronne périurbaine est largement déconnectée du lieu d'emploi ou de la distance à parcourir entre le domicile et ce pôle; d'autre part, même lorsque les périurbains fréquentent quotidiennement, dans le cadre de leur travail, le pôle urbain, ou qu'ils en sont proches géographiquement, ils optent majoritairement pour les services privés de base de leur commune de résidence.

22 En revanche, le fait d'avoir déjà résidé dans le pôle urbain a une influence sur certaines pratiques spatiales des périurbains, en favorisant leur mobilité à destination du pôle. C'est le cas des achats en grande surface et de la fréquentation des services basiques, où le test du khi ${ }^{2}$ est significatif à très significatif, alors qu'il est peu significatif, au contraire, pour les achats anomaux. Un examen approfondi des pratiques spatiales liées à la fréquentation des services, selon la localisation du précédent logement, se révèle particulièrement instructif, à double titre.

D'une part, si l'on retient comme fort probable l'hypothèse selon laquelle les périurbains dont le précédent logement était situé à La Roche-sur-Yon utilisaient les services du pôle urbain dans les mêmes proportions que ceux qui continuent d'y résider, soit à plus des 
quatre cinquièmes, on peut alors mesurer l'ampleur du transfert de fréquentation du pôle vers la couronne qui accompagne une mobilité résidentielle de même sens. En effet, le recours aux services privés du pôle urbain est toujours minoritaire pour ces anciens habitants de La Roche-sur-Yon, oscillant entre un cinquième (pharmacie et médecin généraliste) et, au plus, un tiers (coiffeur). On peut donc en conclure que si l'installation en couronne périurbaine n'a guère modifié les habitudes d'achats des néo-résidents périurbains, elle s'est en revanche accompagnée, pour la grande majorité d'entre eux, d'un changement de fréquentation des services privés de base. La distance-temps à parcourir entre le domicile et le service, autrement dit la proximité entre l'offre et la demande, semble donc déterminante pour comprendre les pratiques spatiales des usagers de ces services.

D'autre part, même si la fréquentation des services privés du pôle urbain reste toujours minoritaire quel que soit le lieu de résidence antérieur, le fait d'avoir résidé auparavant dans ce pôle s'accompagne d'un surcroît de fréquentation significatif de ces services. Des trois services considérés, le test $\mathrm{du} \mathrm{khi}^{2}$ est le plus significatif pour le coiffeur et le médecin généraliste, la fréquentation étant la plus discriminée pour ce dernier : si $3 \%$ seulement des habitants de la couronne ont leur généraliste situé à La Roche-sur-Yon parmi ceux qui n'y ont pas résidé antérieurement, ce taux monte à $21 \%$ pour ceux dont le précédent logement était yonnais. Cette observation nuance le rôle déterminant joué par la proximité entre l'offre et la demande, et laisse supposer qu'une autre dimension que la proximité spatiale permet de rendre compte d'une partie de ces pratiques. En effet, de la pharmacie au coiffeur et au médecin généraliste, on passe d'une relation essentiellement basée sur un échange commercial à une relation marquée par une forme d'intrusion dans l'intimité de l'individu, qui touche à la fois au corps et à l'esprit. La relation de confiance devient alors essentielle, ce que traduit l'usage de l'adjectif possessif ( «mon » coiffeur ou «mon" médecin). Aussi, selon l'importance accordée à cette relation et sa solidité, lorsque l'individu s'installe dans le périurbain après une étape résidentielle dans le pôle urbain, si dans la majorité des cas il choisit un nouveau coiffeur et un nouveau médecin parmi ceux de sa nouvelle commune de résidence, dans d'autres cas, il opte pour la fidélité, ce qui implique alors une mobilité dès qu'il recourt à ce service. Quant à la différence de fréquentation entre le coiffeur et le médecin généraliste, avec respectivement un tiers des anciens habitants du pôle urbain qui ont gardé leur coiffeur dans le pôle urbain, contre un cinquième pour le médecin, elle s'explique probablement par une question de temporalité dans le recours au service. On programme à l'avance son rendez-vous chez le coiffeur, ce qui est d'autant plus facile que pour les trois quarts des périurbains ayant résidé auparavant à La Roche-sur-Yon et qui ont gardé leur coiffeur dans cette ville leur lieu d'emploi est situé précisément dans le pôle urbain. En revanche, le recours au médecin généraliste est, le plus souvent, non programmé, d'où l'intérêt d'en choisir un qui soit proche du domicile.

\section{Des pratiques spatiales des habitants du périurbain plus orientées vers la périphérie}

Lorsque les pratiques spatiales des habitants du périurbain de La Roche-sur-Yon sont orientées en majorité vers le pôle urbain, ces pratiques sont plus souvent tournées, au sein de ce pôle, vers les centres commerciaux et les zones d'emploi périphériques, si on les compare aux pratiques des habitants du pôle yonnais. Les tests ${\mathrm{du} \mathrm{khi}^{2}}^{2}$ sont 
notamment très significatifs pour les achats de vêtements et pour l'emploi, où la diversité spatiale de l'offre est très marquée, alors qu'ils sont peu significatifs pour les achats de livres, du fait sans doute d'une offre beaucoup plus limitée en périphérie.

Ainsi, les deux tiers des habitants de la couronne périurbaine de La Roche-sur-Yon fréquentent généralement le centre-ville pour leurs achats de vêtements, contre les trois quarts des Yonnais (tableau 6). La différence devient beaucoup plus importante lorsque l'on observe la fréquentation exclusive de ce cœur de ville: un peu plus du quart seulement des périurbains achètent en général leurs vêtements exclusivement dans le centre, contre pratiquement la moitié des habitants du pôle urbain. Fort logiquement, à cette moindre fréquentation des commerces du centre par les habitants de la couronne correspond une plus grande fréquentation de la polarité des Flâneries situé au nord de la ville, le long de la voie express menant à Nantes : $58 \%$ des périurbains effectuent leurs achats de vêtements aux Flâneries, contre $42 \%$ des Yonnais. Ce centre commercial, composé de grandes surfaces spécialisées ouvrant sur une rue intérieure et couverte, est au cœur d'une polarité périphérique emblématique des nouveaux lieux de vie qui ponctuent la "ville émergente» (Dubois-Taine et Chalas, 1997): il jouxte un hypermarché, de nombreux autres magasins spécialisés, un multiplex cinématographique et sept restaurants. Au total, pour les habitants de la couronne périurbaine, les Flâneries font presque jeu égal avec le centre-ville pour les achats de vêtements (8 points de différence seulement au profit du second espace), ce qui est loin d'être le cas pour les habitants de La Roche-sur-Yon, qui continuent d'accorder une nette préférence aux commerces situés en cœur de ville (33 points de différence). Le comportement d'achat des habitants de la couronne est donc plus tourné que celui des habitants du pôle urbain vers les Flâneries, révélant ainsi leur tendance affirmée à la « périphérisation » de la pratique de la ville.

Tableau 6 : Les lieux d'achat de vêtements à La Roche-sur-Yon par les habitants de l'aire urbaine selon leur appartenance à la couronne périurbaine ou au pôle urbain en 2000 (Le résultat du test de $\mathrm{Khi}^{2}$ à $99 \%$ est très significatif) The shopping places of clothes in La Roche-sur-Yon by inhabitants living in urban area according to their belonging to surrounding suburbs or urban centre in 2000

\begin{tabular}{|c|c|c|}
\hline \multirow{2}{*}{ Lieux d'achat de vêtements à La Roche-sur-Yon } & $\begin{array}{c}\text { Habitants de la } \\
\text { couronne périurbaine }\end{array}$ & $\begin{array}{c}\text { Habitants du pôle urbain } \\
\text { (La Roche-sur-Yon) }\end{array}$ \\
\cline { 2 - 3 } & en \% & en \% \\
\hline Centre-ville & 65,5 & 74,9 \\
dont centre-ville exclusivement & 27,7 & 48,1 \\
Les Flâneries (polarité périphérique) & 57,7 & 41,8 \\
dont Flâneries exclusivement & 20,4 & 15,4 \\
\hline Total répondants & 426 & 474 \\
\hline
\end{tabular}

(Source : enquête auprès d'un échantillon de 1000 habitants de l'aire urbaine de La Roche-sur-Yon, dont 475 de la couronne périurbaine, 2000)

Cette "périphérisation » se retrouve également au niveau de l'emploi. Près de la moitié (44\% exactement) des périurbains qui viennent travailler à La Roche-sur-Yon ont leur lieu d'emploi situé dans l'un des cinq grands pôles d'activités périphériques ${ }^{10}$, alors que cette proportion n'atteint que le quart pour les habitants du pôle urbain. Ces pôles d'emploi sont sur-représentés pour les habitants de la couronne, dans la mesure où leur 
part dans l'emploi total de La Roche-sur-Yon est d'environ 38 \% en 1999. En revanche, le centre-ville est sous-représenté comme lieu d'emploi: parmi les périurbains dont l'emploi est situé à La Roche-sur-Yon, un cinquième seulement travaille dans le centre, contre plus du tiers pour les habitants du pôle urbain (35\%), alors que cet espace central concentre un quart de l'emploi total de ce pôle.

\section{Conclusion}

Le système urbain, mais aussi la réflexion sur la ville, doivent pleinement intégrer la dimension périurbaine, car la dynamique de périurbanisation s'accompagne de l'émergence de nouveaux comportements et d'une pratique différenciée de l'espace urbain. À la diffusion centrifuge de l'habitat correspond, en quelque sorte, une "périphérisation» des modes de vie, car au développement de l'habitat dans les couronnes périurbaines répond la cristallisation d'une partie de l'activité et du commerce aux franges des pôles urbains, selon une logique de reconcentration à l'origine de polarités périphériques. Or, cette logique est inductrice, à son tour, de localisations résidentielles sur le pourtour des unités urbaines (Jaillet et Jalabert, 1982 ; Dezert et al., 1991), révélant combien les dynamiques résidentielle et économique des aires urbaines, au-delà d'un antagonisme plus apparent que réel, s'auto-alimentent. Il y a bien complémentarité, plus qu'opposition, entre ces deux processus, qui reconfigurent le système urbain en lui offrent une nouvelle cohérence.

Par ailleurs, cette moindre propension à fréquenter le centre-ville lorsque l'on réside en zone périurbaine, au profit des polarités périphériques, va de pair avec un usage quasiexclusif de l'automobile comme moyen de déplacement, dans la mesure où ces polarités, $\mathrm{du}$ fait de leur situation et de leur conception, sont accessibles essentiellement par la voiture. Le cas de La Roche-sur-Yon est, de ce point de vue, tout à fait exemplaire. D'une part, la multi-motorisation est devenue la règle au sein de la population de la couronne périurbaine : $70 \%$ des ménages enquêtés possèdent deux voitures ou plus, contre $45 \%$ pour les ménages du pôle urbain. D'autre part, l'usage exclusif de la voiture s'impose dans les déplacements urbains: $99 \%$ des habitants de la couronne périurbaine utilisent généralement la voiture lorsqu'ils effectuent un déplacement dans la ville de La Rochesur-Yon, contre 80 \% des habitants du pôle urbain. Ce double constat, même s'il demande à être validé par d'autres observations, limite singulièrement la portée d'action de toute politique publique qui viserait à restreindre l'usage de l'automobile dans la ville. En effet, dans ce nouvel état du système urbain où la dilution l'emporte sur la finitude et la densité, le développement de la mobilité et de l'usage de l'automobile sont indissociables, car ils constituent une des composantes des transformations du système urbain à travers ses différentes dimensions, économique, sociale et spatiale. 


\section{BIBLIOGRAPHIE}

Allain (R.), 1992. - La maison et la ville en Bretagne, Thèse de Doctorat d'État, Université de Rennes II, $1348 \mathrm{p}$.

AsCHER (F.), 1995. - Métapolis ou l'avenir des villes, Paris, Odile Jacob, 345 p.

Berger (M.), Fruit (J.-P.), Plet (F.), Robic (M.-C.), 1980. - « Rurbanisation et analyse des espaces ruraux péri-urbains ", L'Espace géographique, $\mathrm{n}^{\circ}$ 4, p. 303-313.

BERGER (M.), 1990. - « Les périurbains d'île-de-France : stratégies de localisation », dans BONVALET (C.), FRIBOURG (A.-M.) (dir.), Stratégies résidentielles, Paris, Ined, p. 369-383.

BOURDIEU (P.), 1990. - « Un placement de père de famille. La maison individuelle : spécificité du produit et logique du champ de production ", Actes de la recherche en sciences sociales, $\mathrm{n}^{\circ}$ 81-82, $\mathrm{p}$. 6-33.

CHAPUIS R., 1995. - «L'espace périurbain. Une problématique à travers le cas bourguignon », L'information géographique, $\mathrm{n}^{\circ}$ 59, p. 113-125.

CHOffel (P.), 2000. - « Les transformations des espaces urbains dans les années 1990 : une périurbanisation au ralenti ", Regards sur l'actualité, p. 15-28.

CROIX (N.), RENARD (J.), 1985. - « Recherches préliminaires sur les populations et les espaces périurbains nantais », dans COLLECTIF, Les périphéries urbaines, Caen, Presses universitaires de Caen, p. 33-44.

DESSE (R.-P.), 2001. - Le nouveau commerce urbain. Dynamiques spatiales et stratégies des acteurs, Rennes, PUR, $198 \mathrm{p}$.

DeZert (B.), METTON (A.), STEINBERG (J.), 1991. - La périurbanisation en France, Paris, SEDES, 226 p.

Dubois-Taine (G.), Chalas (Y.) (dir.), 1997. - La Ville émergente, La Tour-d'Aigues, L’Aube, 285 p.

FRIBOURG (A.-M.), 1998. - «Évolution des politiques du logement depuis 1950 », dans SÉGAUD (M.), Bonvalet (C.), Brun (J.) (dir.), Logement et habitat, l'état des savoirs, Paris, La Découverte, p. 223-230.

HAUMONT (N.), LÉVY (J.-P.) (dir.), 1998. - La ville éclatée. Quartiers et peuplement, Paris, L’Harmattan, $261 \mathrm{p}$.

HÉRIN (R.), 1985. - « Les espaces périurbains : des projets et des systèmes de valeurs inscrits dans l'habitat et les pratiques sociales ", dans COLLECTIF, Les périphéries urbaines, Caen, Presses universitaires de Caen, p. 140-143.

Heugas-DarRasPen (H.), 1994. - Le financement du logement en France, Paris, La documentation Française, $181 \mathrm{p}$.

JAillet (M.-C.), JALABERT (G.), 1982. - « Politique urbaine et logement : la production d'espace périphérique ", L'Espace géographique, $\mathrm{n}^{\circ}$ 4, p. 293-306.

JEAN (Y.), CALENGE (C.), 1997. - « Espaces périrubains : au-delà de la ville et de la campagne ? (Problématique à partir d'exemples pris dans le Centre-Ouest) », Annales de Géographie, n 596, p. 389-413. 
JULIEN (P.), 2001. - « Les grandes villes françaises étendent leur influence », Insee Première, n $^{\circ}$ 766, $4 \mathrm{p}$.

LAINÉ (F.), 2000. - «Péri-urbanisation des activités économiques et mouvements d'emploi des établissements », dans Matté (M.-F.), Pumain (D.) (dir.), Données urbaines 3, Paris, Anthropos, p. 251-260.

LE GuirRiec (P.), 1997. - « Mobilité et identité dans la périphérie brestoise », Dubois-TAINe (G.), C HALAS (Y.) (dir.), La ville émergente, La Tour-d'Aigue, L'Aube, p. 78-85.

LE JEANNIC (T.), 1996. - « Une nouvelle approche territoriale de la ville », Économie et Statistique, $\mathrm{n}^{\circ}$ 294-295, p. 25-45.

-, 1997. - «Trente ans de périurbanisation : extension et dilution des villes », Économie et Statistique, $\mathrm{n}^{\circ} 307$, p. 21-41.

MADORÉ (F.), 1998. - « La demande en logements : de la pavillonnarisation de l'espace périurbain à la revalorisation des quartiers anciens ", dans BeRTRAND (J.-R.), CHEVALIER (J.) (dir.), Logement et habitat dans les villes européennes, Paris, L'Harmattan, p. 165-181.

MAy (N.), Veltz (P.), LANDRieu (J.), SPECTOR (T.), 1998. - La ville éclatée, La Tour-d'Aigues, L'Aube, $354 \mathrm{p}$.

ORfEuIL (J.-P.), 1995. - « Mobilité : les territoires du quotidien », dans AscheR (F.) (dir.) Le logement en questions, La Tour-d'Aigues, L'Aube, p. 171-188.

PiHET (C.), 1985. - « À propos d'une enquête : l'espace social des néo-résidents du périurbain à l'ouest d'Angers ", dans COLLECTIF, Les périphéries urbaines, Caen, Presses universitaires de Caen, p. 233-236.

SCHMITT (B.), PERRIER-CORNET (P.), 1998. - Les campagnes et leurs villes, Paris, Insee, coll. « Contours et caractères ", 203 p.

TAFFIN (C.), 1985. - « Accession à la propriété et "rurbanisation" ", Économie et Statistique, n 175, p. 55-67.

TALвот (J.), 2001. - « Les déplacements domicile-travail. De plus en plus d'actifs travaillent loin de chez eux ", Insee Première, $n^{\circ} 767,4$ p.

VORMS (B.), 1998. - « Les politiques d'encouragement à l'accession à la propriété ", dans SÉGAUD (M.), BonVAlet (C.), BRUn (J.), Logement et habitat, l'état des savoirs, Logement et habitat, l'état des savoirs, Paris, La Découverte, p. 210-219.

\section{NOTES}

1. On peut se reporter en particulier à l'ouvrage Les périphéries urbaines, qui fait la synthèse, au milieu des années quatre-vingt, des recherches conduites sur le périurbain en France. Certaines contributions abordent plus spécifiquement la question de la mobilité quotidienne des périurbains, notamment celles de N. Croix et J. Renard (1985), de R. Hérin (1985) ou encore de C. Pihet (1985).

2. Les 1000 enquêtes ont été passées au cours des mois de mars et avril 2000 par treize enquêteurs étudiants du Deug 2 de géographie au centre universitaire de La Roche-sur-Yon, qui dépend de l'Université de Nantes.

3. Les données du recensement de 1999 n'étaient pas encore disponibles lorsque l'enquête a été réalisée en mars et avril 2000. 
4. Quelques extraits rendent compte de cette convergence d'appréciation sur le fait périurbain. Pour C. Taffin, (1985) «le développement des zones périurbaines n'est pas une nouvelle forme d'exode rural, mais bien le fait de la déconcentration des zones urbaines ». Pour R Chapuis, (1995) « la périurbanisation est une nouvelle forme de l'expansion urbaine, mais qui n'est plus, comme la banlieue traditionnelle, morphologiquement rattachée à la ville ». Pour Y. Jean et C. Calenge, (1997) il ne fait guère de doute que l'espace périurbain a «la qualité de sous-ensemble de la ville ». Enfin, pour T. Le Jeannic, (1997) « le phénomène de périurbanisation apparaît ainsi bien in fine comme un phénomène local de débordement de la population d'un centre sur sa périphérie ».

5. Les communes multipolarisées sont des communes rurales ou des unités urbaines situées hors des aires urbaines, dont au moins $40 \%$ de la population résidente ayant un emploi travaille dans plusieurs aires urbaines, sans atteindre ce seuil avec une seule d'entre elles, et qui forment avec elles un ensemble d'un seul tenant. Avec les couronnes périurbaines, elles forment les communes périurbaines.

6. Le rural isolé est formé de l'ensemble des communes rurales et des unités urbaines appartenant à l'espace à dominante rurale et n'étant ni pôle rural, ni sous faible influence urbaine, ni périphérie des pôles ruraux. Les pôles ruraux sont des communes rurales ou des unités urbaines appartenant à l'espace à dominante rurale, offrant de 2000 à 5000 emplois et dont le nombre d'emplois offerts est supérieur ou égal au nombre d'actifs résidents. Le rural sous faible influence urbaine est constitué de l'ensemble des communes rurales et des unités urbaines appartenant à l'espace à dominante rurale, qui ne sont pas pôle rural et dont $20 \%$ ou plus des actifs résidents travaillent dans des aires urbaines. Quant à la périphérie des pôles ruraux, elle est constituée des communes rurales ou des unités urbaines appartenant à l'espace à dominante rurale, n'étant ni pôle rural, ni sous faible influence urbaine, et dont $20 \%$ ou plus des actifs résidents travaillent dans les pôles ruraux.

7. Il est en effet constitué d'une ville isolée, soit d'une seule commune, selon la nomenclature des unités urbaines de l'Insee.

8. Les tris croisés effectués pour mesurer les pratiques spatiales retenues (l'emploi, les achats et les services privés de base) selon le lieu de résidence (pôle urbain ou couronne périurbaine) sont tous fiables. Le résultat du test de $\mathrm{Khi}^{2}$ à $99 \%$ est très significatif pour les achats en grande surface et de vêtements, pour le lieu d'emploi, pour la fréquentation du salon de coiffure, de la pharmacie et du médecin généraliste. Il est significatif pour les achats de livres.

9. Le terme " yonnais » fait toujours référence au pôle urbain de La Roche-sur-Yon. Il désigne soit les habitants de ce pôle (les Yonnais), soit des équipements (exemple, les hypermarchés et les supermarchés yonnais).

10. Ces cinq pôles périphériques correspondent aux trois concentrations de zones d'activités économiques, soit la zone Nord (Acti-Nord et les Flâneries), la zone Sud (Acti-Sud, Les Jaulnières et Belle-Place) et la zone des Ajoncs au nord-est, et aux deux centres hospitaliers, à savoir le Centre hospitalier départemental (CHD) au nord-est et le Centre hospitalier sud (CHS) au sud.

\section{RÉSUMÉS}

Cet article insiste sur les conséquences, en termes de schéma de mobilités quotidiennes des ménages, sur un contraste majeur dans l'évolution de l'organisation urbaine en France. En effet, on observe un phénomène de dissociation relative entre d'une part la croissance démographique 
centrifuge des aires urbaines et, d'autre part, le maintien d'une localisation centrale de l'emploi et du commerce au sein de ces entités. Cette réflexion sera développée à partir d'une étude de cas fondée sur l'observation du fonctionnement d'une aire urbaine de taille moyenne, celle de La Roche-sur-Yon en Vendée (94000 habitants). Fort logiquement, le développement de la dynamique de périurbanisation s'accompagne de l'émergence de nouvelles pratiques spatiales dont certaines, liées en particulier à la fréquentation des commerces ou des lieux d'emploi, sont fortement orientées vers le pôle urbain de La Roche-sur-Yon, alors que d'autres sont marquées au contraire par la fréquentation des services situés dans les communes de la couronne périurbaine, et plus particulièrement ceux de la commune de résidence. Par ailleurs, ces pratiques citadines mises en œuvre par la population périurbaine s'accompagnent de nouveaux comportements et d'une fréquentation différenciée de l'espace urbain, plus portée vers les polarités périphériques que vers le cœur de la ville.

This paper joints out one of the major contrast of present evolution of urban organisation in France: the daily mobilities of families. The contrast is bare on a phenomenon of relative separation between, on the one hand, the demographic growth in urban areas marked by the vigour of the suburbanization process and on the other hand, the job and trade localization within these entities (their concentration in urban poles has never failed). This reflection will be developed from a case study based on the observations of a middle-sized urban area, called La Roche-sur-Yon in Vendee (94000 inhabitants). Very logically, the development of the suburbanization dynamic is accompanied by the emergence of new spatial practices some of which, particularly closed to the shop-going or the job-going localization (national employment agency), are mainly directed towards the urban pole of La Roche-sur-Yon. Others are really marked, at the opposite, by the frequenting of offices based in the districts of La Roche-sur-Yon suburbs, and more precisely those of the residential suburbs. Moreover, these urban practices created by the suburban population are linked with new behaviours and a differentiated frequenting of the urban space, much more orientated towards the outlying polarities than towards the center of the city.

\section{INDEX}

Mots-clés : emploi, mode de vie, périurbanisation

Keywords : employment, suburbanization, ways of life

Index géographique : France, La-Roche-sur-Yon

\section{AUTEUR}

\section{FRANÇOIS MADORÉ}

Institut de géographie (IGARUN) - Université de Nantes, ESO (Espaces géographiques et sociétés)UMR 6590 - CNRS, françois.madore@humana.univ-nantes.fr 